\title{
The Database Administrator's Readiness to Migrate Database in the Cloud
}

\author{
Arwa Aleryani \\ Associate Prof, Independent Researcher \\ arwa.aleryani@gmail.com \\ DOI: 10.29322/IJSRP.11.08.2021.p11668 \\ http://dx.doi.org/10.29322/IJSRP.11.08.2021.p11668
}

\begin{abstract}
Many companies and businesses - large and small- intend to migrate databases to the cloud in order to take the advantages and benefits of this transition. The current research aims to study and examine the readiness of the database administrator to migrate and manage the database in the cloud. The research aims to examine and find out the tasks that will be added to the responsibilities of the database administrator that she/he must perform regards this transition. The current research will also address the strengths that the database administrator must possess before starting the project, and the potential weaknesses that he/she must work to over them. The research will also address the opportunities (tangible and intangible) that the database administrator will gain, and at the same time, the challenges and threats that she/he has to face and work to mitigate their impact on the quality of database performance in the cloud.
\end{abstract}

Index Terms- DBAs, Cloud Databases, Readiness.

\section{INTRODUCTION}

$\mathrm{C}$ loud computing has changed the way that management information systems (MIS) services are provided, and the companies' employees conduct business and perform key tasks (Mew \& Money). Outsourcing, lean, and flexible management, change management, and cost reduction are all gaining popularity as businesses confront increasingly difficult economic conditions. This is because cloud computing is geared towards saving money and dealing with unforeseen changes. It for example, has the potential to turn information technology, which has traditionally been viewed as a cost center, into a source of cost savings and increased flexibility and agility for companies (Sawas \& Watfa, 2015).

Cloud computing sometimes called on-demand computing is ascending quickly along with its data-center growing at a remarkable standard. It appears as a unique and attractive computing pattern that intents to provide customized, decisive, and quality of service guaranteed effective computing situations (Al-Shafi \& Bahar).

People are now using online way for dealing with their daily transactions and shopping to save time as a result of technological advancements. Companies are now able to think of the fastest way to conduct business online due to this shift in business. There was a time when programs had to be installed on computers in order to access the company database, yet today's employees do not engage their time in more productive tasks"

the time to do so. They prefer to use a cloud database since it allows them to quickly access the information saved in their database.

Cloud Database Service Providers (DBaaS) relieve customers of the burden of making quick database modifications. On the other hand, they give scalability during peak times, ensuring that a corporation does not underperform. Customers, employees, and corporate authorities may receive the configuration they want from anywhere, at any time. Making it very strong technology organizations ( $\mathrm{Al}$ Sheheri, 2013).

Migration to the cloud is not a one-size-fits-all endeavor. Organizations must develop intelligent business cases by outlining key objectives and then effectively communicating the expected advantages of this essential digital transformation project.

The aim of this research is to answer two questions: What are the new DBAs' responsibilities and skills to migrating and managing the database on the cloud? How is the DBAs' readiness to migrating and managing the database on the cloud? This will be done by The Qualitative method and SWOT analysis.

The current study is organized into four parts; the first part is the literature studies, the second part is the research methodology, the third part is the analysis and discussion, and the final is the conclusion.

\section{Literature Studies}




\section{A. Cloud computing}

Cloud computing has changed the way that management information systems (MIS) services are provided and their employees conduct business and perform key tasks. This emerging cloud paradigm has significantly impacted the industry providing MIS database development services, from employee roles and competencies to the provision of services by development contractors (Lionel Mew). Latest technology such as cloud computing promises to transform information technology, which has always been seen as a cost center, into a source for saving money and driving flexibility and agility for businesses (Mohamed S. Sawas).

Cloud computing has been the most adaptable technology in recent times, and the database has also moved to cloud computing now, so we will look into the details of a database as a service and its functioning (Al Shehri, 2013). There are many features of the cloud; Resource pooling, On-Demand self-service, Easy Maintenance, Large Network Access, Availability, Automatic System, Economical, Security, Pay as you go, Measured service (Chaubey \& Nanda,2020), (Radack, 2012), (Bhatti \& Rad).

\section{B. Cloud Database}

Database as a Service (DBaaS) is an architectural and operational strategy that allows IT companies to supply database functionality to one or more customers as a service. Database products on the cloud can be used in two scenarios to meet an organization's database needs. First one is a single large organisation that has many individual databases which can be migrated to a private cloud and second one, is an outsourcing the data management needs of small and medium organisations to a public cloud provider, who caters to multiple small and medium businesses. (Malliga, 2012).

Al Shehri (Al Shehri, 2013) studied the database as a service. He discussed the challenges that are facing moving databases to the cloud. The study listed some of the cloud database services for example; choosing the best DBaaS, data Sizing, portability, transaction capabilities, configuration, database accessibility, certification and accreditation, data integrity, security, and storage location. Some of the main challenges facing cloud databases have been mentioned such as internet speed, query, and transactional workloads, multitenancy, elastic scalability, privacy.

\section{Cloud Database Administrators}

Many articles and studies have addressed the new roles and responsibilities of the cloud databases administrators (DBAs).

Database administrators are the driving force behind the entire cloud database management system, as they are in charge of providing cloud users with databases as a service (Alam \& Shakil, 2013). Wendy Neu and the co-authors (Neu et al., 2019) listed DBA tasks that are changed because of moving database to cloud including provisioning, ETL, data ingestion, and data export, Backups and snapshots, access, maintenance of development and testing environments, logs, monitoring and notifications, performance optimization, handling high availability.

Burnham (Burnham, 2018) listed in his report what the experts say about the DBAs new tasks as follows:

- DBA can be a partner with the business, Jim Johnson, senior VP at Robert Half Technology says, "The reality is now that the business understands the value of data, the DBA needs to be able to move at cloud speed". He adds "They're having to be more in tune with the business and act as more than just the database administrator - they need to be the data experts." Moreover, Lane Greever, senior vice president at Modis says "Some organizations are encouraging this partnership by integrating DBAs with the data science team. Especially in larger organizations, the DBA is a very tenured role. With that comes domain and industry knowledge".

- DBA can create new value, Jim Johnson says "Cloud is taking them away from some of the things they are used to doing, whether it's being on-call 24/7 or managing backups" Johnson continues "This shift in responsibilities gives DBAs the opportunity to be more involved in helping the business leverage the data they have. DBAs need to focus on enabling the business and coming to them with ideas." On the other hand, Angove says "This might mean taking a more proactive role in problem-solving.

- DBA learns more about everything, Johnson says "Because the database administrator's role is evolving beyond its traditional responsibilities and confines, DBAs need to take a more holistic view of their skillset. DBAs need to look at the broader picture and understand that they're looking at solutions rather than just databases," he says. "They need to take off the blinders and look at the whole impact that these old databases have on the business and think about how they can help their company better use that data."

- Angove says, "Staying current also means pursuing relevant certifications, DBAs should ensure their traditional database certifications are up to date, and they should strongly consider relevant cloud certifications, such as AWS or Microsoft Azure". He adds "Don't remain siloed in the traditional database administrator role. Gain an understanding of BI, cloud, and securityeverything that touches the database.

- DBA should not lock themselves into a title, Greever says "While the word "cloud" might worry database administrators, look at it as a positive. Cloud is forcing DBAs to move to other parts of the business, align with the business side of the house, and it's forcing them to get outside of their comfort zone". "Because DBAs are getting this new experience and learning new skills when it is time to move onward or upward, do not limit yourself to another database administrator job" he adds. 
Kelly (Kelly,2020)) discussed in his article the responsibilities that DBAs have on traditional databases and how the evolution of the cloud has changed the way DBAs are approaching their work. The author listed some of the DBAs responsibilities and showed how they have been changed as follows:

Capacity Planning: Traditionally, database capacity preparation entailed allocating server resources to handle peak loads; this is not only more expensive but also less versatile. Cloud-hosted databases, on the other hand, are scalable, meaning that their storage and computing power can be dynamically and automatically scaled to meet changing demands.

Database Backup and Recovery: Traditional database backup methods include storing and preserving physical backup devices like tapes. The cloud provides simpler and more long-lasting storage solutions that keep multiple copies of backup data in multiple physical locations automatically. Backups and recovery can be scripted and automated since there are no physical storage devices for the DBA to handle.

Choosing the Optimal Database Solution: Cloud platforms provide new database options for teams of designers, including selfmanaged and completely managed relational, NoSQL, key-value pair, document, in-memory, columnar, graph, time-series, and other databases.

Designing for the Workload: With the move to managed cloud services, database administrators don't have to spend as much time on repetitive maintenance activities like patching, updating, and installing database engines. They will now provide advice to application developers on which database service to use for a given workload, as well as perform a smaller number of administrative tasks.

Continued Training and Education: To keep current with business developments and technologies, database professionals can take advantage of continuing education and accreditation opportunities.

Mckendrick (McKendrick, 2019)) had also discussed how the DBAs jobs have been changed with moving database to cloud. Cloud means a number of changes to DBAs' jobs, including the following:

- The growing value of cloud-based data and databases frees DBAs from mundane, day-to-day activities, allowing them to spend more time directly advising company leaders, managers, and consumers about how data can be used to solve business problems.

- For today's data professionals, emerging positions such as enterprise data architects, data stewards, and data engineers offer new career paths. Furthermore, the cloud facilitates in which developers and development teams collaborate to produce highquality software at the speed required by the business.

- Greater capabilities necessarily require a greater focus on data, regardless of format or origin. Modern applications, such as social media, finance, and fantasy gaming apps, are better suited for cloud-based database systems.

- With the cloud, today's databases are less likely to serve just a small number of internal users and are more likely to serve audiences outside of corporate firewalls. The DBA's function is changing from that of a single, skilled database operator to that of a maestro who can organise a symphony of data environments.

- When businesses strive to succeed in this modern era, they rely heavily on managers and experts to assist them in making the transition to the cloud. This requires DBAs to have a thorough understanding of current structures and application specifications.

- The most common type of cloud deployment these days is "accidental hybrid environments," in which various groups choose different cloud systems based on their own requirements, resulting in an organization supporting a diverse collection of clouds. DBAs are being called on more and more to step up and provide clarification and guidance in the face of cloud uncertainty.

Norris (Norris, 2017a) discusses the typical database administrator, who is a person responsible for activities that are directly or indirectly connected to the lifecycle of databases in an organization. His/her responsibilities include:

- Designing, installing, and configuring databases.

- General maintenance: hardware, upgrades, backups, troubleshooting, recovery.

- Migration, security, performance tuning and monitoring of databases.

- Developer assistance.

Moreover, Norris discussed how the cloud has transformed the function of most technology-based businesses. The change has brought numerous benefits to the way companies develop and deploy applications, but it has also forced most engineers, including DBAs, to restructure their jobs. Some of the skills necessary for being a successful DBAs include:

- The service provider provides the majority of cloud services. As a result, it is important that the cloud DBAs have a thorough understanding of the services that the company uses. This skill set involves being well-versed in the costs associated with managing data infrastructure and architecting systems in accordance with the company's needs.

- When it comes to confidential and important data, information is stored in the cloud which leads to a range of challenges. The DBAs must be able to prepare for and execute scenarios so that any malfunction in the database does not disrupt the company's ability to operate. This duty necessitates a thorough and nuanced understanding of the importance of the company's data, as well as taking the necessary steps to ensure that this value is preserved when data-related technology fails. 
- Although most public cloud vendors provide security mechanisms and reasonably secure services, data security is still a top concern. Protecting sensitive information across highly distributed infrastructures can be a challenge, and the DBAs need to explore possible internal and external threat sources and comply with corporate security guidelines.

The same author (Norris 2017b) discussed in her second article the skills needed for DBAs' new roles when the database moved to the cloud. Whether the organization adopts an Infrastructure-as-a-Service (IaaS), Database-as-a-Service (DBaaS), or a hybrid architecture, the DBA tasks have shifted significantly:

- In the IaaS model, the DBA must perform all of the tasks that a conventional database needs but does not have to worry about provisioning hardware resources.

- The DBaaS model simplifies activities such as installation, setup, patching, backup and recovery, and so on. Moreover, the DBaaS model allows the DBA to focus on higher-level tasks, such as application and database creation. The DBA in a hybrid architecture must consider how all of these capabilities interact in a single device. The author discussed the following responsibilities:

- Estimating database resources and planning capability has been a central DBA activity since the beginning of database engines. DBAs must comprehend the system's target for which the database resource is needed. However, these planning tasks in the IaaS model are somewhat similar to those in on-premises, virtualized environments.

- Understanding how data can be migrated and preparing end-to-end data migration at the data level has become very relevant for DBAs with rapidly evolving market needs and increasing resource requirements to manage development: what is the target platform? what are the database software changes? what infrastructure needs to be provisioned? and so on. DBAs must understand how the new platform works and evaluate various database software and technology choices.

- DBAs collaborate with database development teams to investigate performance problems and propose tuning solutions such as partitioning, indexing, and properly distributing data on logical storage. Cloud DBAs get the provider's bundled monitoring and tuning tools when the database is running in an IaaS environment, but they do not meet all of the tuning concerns.

- DBAs in an IaaS database would follow the same backup and recovery procedures as they would in on-premises data centers. However, backup failure is still a possibility, causing data recovery problems and generally lengthening the time it takes to recover.

- Most companies tend to keep certain database components on-premises, so a hybrid architecture would be preferred. DBAs with experience introducing and monitoring both IaaS and DBaaS will be at the forefront of assisting their companies' vertical and horizontal expansion. The DBA is responsible for determining whether a hybrid solution is a good match for the company's IT growth strategy. They must gain a thorough understanding of the implementation and monitoring discrepancies between different IaaS and DBaaS providers. Prior to introducing a hybrid deployment, DBAs must conduct security and cost assessments of databases. DBAs must be familiar with both IaaS and DBaaS features, as well as the expense and feasibility of handling the organization's databases in a hybrid architecture.

\section{RESEARCH METHODOLOGY}

The qualitative method has been used for this research. It is primarily exploratory research. It is used to gain an understanding of underlying reasons, opinions, and motivations. It provides insights into the problem and helps to develop ideas or hypotheses for potential quantitative research (DeFranzo, 2011).

The current research aims to find out what moving databases, to the cloud, will add to DBAs' tasks and what will it take out from them. The researcher searched in the literature studies and official websites to find about the cloud database administration (CDBA) new tasks and new skills.

Moreover, SWOT analysis has be done to evaluate the DBAs' readiness with regards to this movement in terms of their strengths and weaknesses and simultaneously, the opportunities and threats.

Measuring readiness is a systematic analysis of an organization's ability to undertake a transformational process or change. A readiness assessment identifies the potential challenges that might arise when implementing new procedures, or processes within a current organizational context (HRSA, 2011). In order to measure the DBAs readiness, the author conducts a SWOT analysis after determining the main new tasks and new required skills.

A SWOT, which stands for strengths, weaknesses, opportunities and threats, can be used to make an analysis for employees entail a self-evaluation of a person's strengths and weaknesses (Suttle). 


\section{A. Research Design}

The research depends on descriptive and qualitative approaches, first literature reviews are studied to understand the new tasks and the new required skills. From this information, the author conducts a SWOT analysis to find out the strengths, weaknesses of the DBAs as well as their opportunities and threats. The aim is to evaluate the DBAs' readiness to move the database to the cloud.

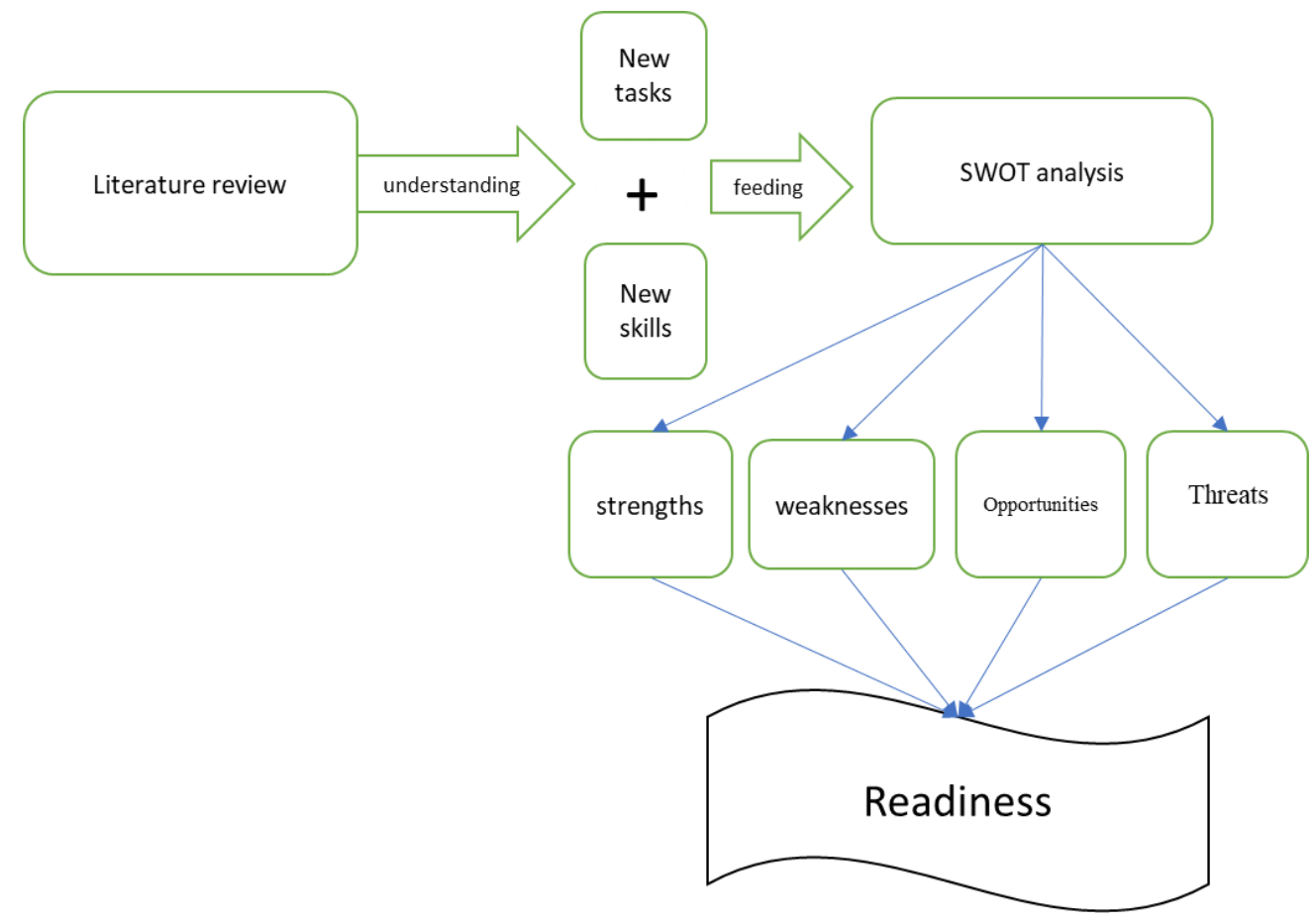

Figure 1: Research Design

\section{B. Research problem}

Migration to the cloud is not a one-size-fits-all endeavor. Organizations must develop intelligent business cases by outlining key objectives and then effectively communicating the expected advantages of this essential digital transformation project.

The decision to move databases to the cloud is an important decision, and it must have all the necessary information to improve the decision. This decision is divided into sub-decisions, including what type of platform is suitable for hosting the company database? What is the transition strategy? Who is the right provider? How to organize a proper control of the database;

How to organize the confidentiality of the assets of the system; How to ensure that what is paid only for what is used and that the cost is not more than expected.

In order to reach a sound decision, the company must have a database administrator who understands the new tasks and has the ability to perform them. So, the current research investigates these tasks and finds out the required skills that make DBAs ready for their new task.

Readiness means the state of being fully prepared for something, in the current research, the readiness of the DBAs to migrate and manage databases in the cloud will be addressed.

\section{Research questions}

The research questions are:

1. What are the new DBAs' responsibilities and skills to migrate and manage the database on the cloud?

2. What is the DBAs' readiness to migrate and manage the database on the cloud? 


\section{ANALYSIS AND DISCUSSION}

The researcher of the current research has derived from the above literature studies that the DBAs require/should have the following new tasks and the new required skills. The tasks that are not anymore on the DBAs responsibilities are capacity planning, database backup, and recovery, and maintenance activities. Now they are performing a smaller number of administrative tasks. On the other hand, DBAs must continue training and education to keep current with business developments and technologies. Now they have the position as "Data Professional" and they have a greater focus on data to advise the company leaders, managers, and consumers about how data can be used to solve business problems. It is still their top concern and responsibility to explore possible internal and external threat sources and comply with corporate security guidelines.

To answer the first question; "What are the new DBAs' responsibilities and skills to migrating and managing the database on the cloud?

Before moving the company's database to the cloud, many tasks should be taken as mentioned by (SADA.COM ) including DBAs assess the current data environment and the skills \& expertise of their internal team. Also, they have to clean the data and rebuild their database schema. It requires a database administrator to have a very good understanding of cloud computing, its advantages and its limitations. Furthermore, a good understanding of service providers and the ability to compare between them and choose what suits the privacy of the company's database. The most important and necessary experiences are understanding the payment entitlements, adjusting payments according to needs, and not exceeding the prepared budget.

\section{A. The skills required to achieve the new responsibility}

Selecting the right cloud provider: Cloud DBAs should understand how to select the right cloud provider, look to the follows: Certifications \& Standards, Technologies \& Service Roadmap, Data Security, Data Governance and Business policies, Service Dependencies \& Partnerships, Contracts, Commercials \& SLAs, Reliability \& Performance, Migration Support, Vendor Lock-in \& Exit Planning, Business health \& Company profile.

There are various approaches for determining a service provider's reliability. First, DBAs should compare the service provider's performance over the last 6-12 months against their SLAs. Then the DBAs should check that the monitoring and reporting tools available are adequate and that they can be integrated into your overall management and reporting systems. The service providers should have procedures and plans in place that detail how they intend to communicate with customers during outages, including timeliness, prioritization, and severity level assessment of issues.

Understanding business terms: There are numerous terms addressed in the cloud module that DBAs should be familiar with, but significant considerations include contractual and service governance, particularly the extent to which the provider can amend the terms of service or contract unilaterally. What are the policies on contract renewals and notice periods for exit or modification? What kind of insurance policies, guarantees, and fines does a company have? What consequences and repercussions are included? As well as any caveats, what is the provider's willingness to subject their company to auditing operations and policy compliance?

Understanding data policies and protection: DBAs should examine a provider's security and data management policies, especially those that pertain to data privacy legislation. And they should ascertain that data access, data location and jurisdiction, confidentiality, and usage/ownership rights are all adequately protected. Also, they have to examine your backup and disaster recovery plans, and they have to examine data conversion policies to see how transportable data might be affected if you decide to depart.

Selecting cloud platform: The decision to move the database to the cloud needs a careful understanding of the benefits and challenges of the cloud. This is the first step, and the DBAs should make sure they understand their business and its needs, because there are many options where the cloud providers offer, they should select the best platform that fits the nature of the business. Moreover, DBAs should have the ability to securely acquire, replicate, and assure the availability of data beyond the relational database management systems within the local corporate data center. DBAs should have the experience in introducing and monitoring both IaaS and DbaaS to assist the companies' vertical and horizontal expansion, they have to determine whether a hybrid solution is a good match for the company's IT growth strategy and understanding the implementation and monitoring discrepancies between different IaaS and DbaaS providers. Prior to introducing a hybrid deployment, DBAs must conduct security and cost assessments of databases.

Selecting the best cloud strategy: There are several cloud strategies, so the could DBAs should have the ability to select the proper type of cloud service that reflects the company's needs.

Determine required cloud services: Cloud providers offer the service as required from the client. The DBAs should understand the costs associated with managing data infrastructure and architecting systems in accordance with the business's needs. They must be sure that they pay only for what their business needs not more.

Following that, the DBAs will be ready to step into the cloud by taking the following steps:

Manage cloud deployment: Cloud DBAs are expected to build and maintain highly distributed data pipelines that are highperformance, in a context where the definition of high-performance might change overnight (because, for example, a competitor might be able to perform the same operation faster). For this task, DBAs need to be skilful in monitoring the performance of the infrastructure and constantly find ways to make it faster.

Data Migration: Cloud DBAs should understand how the new platform works and evaluate various database software and technology choices.

This publication is licensed under Creative Commons Attribution CC BY

http://dx.doi.org/10.29322/IJSRP.11.08.2021.p11668

WWW.ijsrp.org 
Database Performance and Tuning: Cloud DBAs get the provider's bundled monitoring and tuning tools when the database is running in an IaaS environment, but they do not meet all of the tuning concerns. DBAs should expect an improved and integrated management console with DbaaS, which makes tracking and making fast decisions a breeze.

Disaster recovery: DBAs should be familiar with the provider's disaster recovery policies, procedures, and capabilities to meet your data preservation needs. The service agreement must explicitly describe roles and duties, escalation mechanisms, and who bears the burden of evidence. This is critical because DBAs are often in charge of executing some of these processes. They should consider purchasing additional risk insurance if the costs associated with recovery are not covered by the provider's umbrella terms and conditions.

\section{B. SWOT Analysis}

To answer the second question:

How is the DBAs' readiness to migrate and manage the database on the cloud?

The researcher worked on the SWOT analysis to determine the DBAs' readiness. The main elements are strengths, weaknesses of the DBAs in general and the opportunities and threats that might be faced by them as listed in table 1.

\begin{tabular}{|c|c|}
\hline \multicolumn{2}{|c|}{ SWOT analysis to measure the readiness of BDAs to manage the database in the cloud } \\
\hline \multicolumn{2}{|c|}{ Internal strengths and weaknesses of DBAs } \\
\hline DBAs' Strengths & DBAs' weakness \\
\hline $\begin{array}{l}\text { The following strengths might be there for the good } \\
\text { DBAs: } \\
\checkmark \quad \text { Understanding their business and the } \\
\quad \text { company's abilities and the budget. } \\
\checkmark \quad \text { Knowing their database activities and } \\
\text { transactions load deeply. } \\
\checkmark \quad \text { Knowing their clients and their requirements. }\end{array}$ & $\begin{array}{l}\text { The following weaknesses might be there for some } \\
\text { DBAs: } \\
\text { - Understanding the cloud environment and } \\
\text { its requirements. } \\
\text { - Managing the budget for the cloud database } \\
\text { requirements. }\end{array}$ \\
\hline \multicolumn{2}{|c|}{ External Opportunities and threats that are provided to DBAs } \\
\hline Opportunities & Threats \\
\hline $\begin{array}{l}\text { DBAs might gain the following opportunities: } \\
\checkmark \quad \text { Upskills that the DBAs will take to be ready } \\
\text { for their new tasks. } \\
\checkmark \quad \text { A smaller number to be performed of } \\
\text { administrative tasks. } \\
\checkmark \quad \text { Less workload and less time to spend on } \\
\text { repetitive maintenance activities. } \\
\checkmark \quad \text { More time to provide advice to application } \\
\text { developers on which database service to use } \\
\text { for a given workload. } \\
\checkmark \text { More time directly leading and advising } \\
\text { company leaders, managers, and consumers } \\
\text { about how data can be used to solve business } \\
\text { problems. } \\
\text { Gain the new position with the term "Data } \\
\text { Professional" that is capable of cleaning, } \\
\text { selecting, analyzing, and visualizing data. }\end{array}$ & $\begin{array}{l}\text { DBAs might face the following threats: } \\
\text { - Lose full control over the server where their } \\
\text { databases are stored in a cloud. } \\
\text { - Less ability to improve the security of the } \\
\text { company's cloud database. } \\
\text { - The possibility that they will find the } \\
\text { company is paying more than planned. }\end{array}$ \\
\hline
\end{tabular}

Table 1: The SWOT analysis

DBAs will have advanced functions and tasks that are to integrate technology into their data analytics and provide different insights that serve competition and development. Smith listed (Smith, 2015) these technologies as:

Discovery - The data integration landscape is changing, in large measure thanks to the cloud, Virtualization - it will rapidly become a necessity for advanced data integration.

Movement - The movement of data between multiple sources is also critical.

Identification - where does data live, and who owns it? Data identification technologies aim to answer this question and provide a starting point for integration. 
Specificity - data integration solutions will trend toward "tooling that effectively and naturally understands and validates industry coding, provides meaningful data profiling, componentizes processing for reuse, and can handle the sheer volume of business data.

Gravity - New data integrations able to manage and curate this gravity will help companies avoid the problem of data black holes, where information enters but insight never leaves.

On the other hand, DBAs as the data professionals will start using the new technology, for instance, artificial intelligence, machine learning, and natural language processing to get the advanced analytics of their databases.

There's still a learning curve, but self-service BI and data visualization tools are redefining the way businesses leverage all the data they collect into actionable analytics. (Marvin, 2017).

\section{Conclusion}

The researcher can conclude from what was discussed and from the SWOT analysis table that the positive side is more abundant than the negative side.

When the DBAs have a deep understanding of the nature of the company's work, its capabilities and budget, as well as a full understanding of the database, the nature of its activities, and the daily volume of work in it, the company can trust the DBAs' abilities to manage the project of moving the database to the cloud.

However, there are some weaknesses that DBAs may have, but with intensive and focused courses on the concept of cloud services and the working rules with them as well as understanding what are the service providers offer in the market and the ability to decide between them, will overcome the weaknesses.

DBAs will gain many benefits as they will be under the title "Data Professional" instead of "Database Administrator". They will have the time to break free from routine work and use their knowledge and experience to do advanced data analytics and better understand the company's competitive opportunities, as well as will have time to participate in providing advice and plans to improve and develop the company's work.

In terms of the threats that DBAs may face, they can be mitigated through an accurate understanding of these threats, monitoring their possibility, and working to find quick solutions or treatments for them. There is no doubt that this can be done through having clarity of vision of the DBAs on the new environment and the continuing training for a better understanding to work on the cloud.

The new role of DBAs is a "Data Professional" in some companies or "Data scientist" in others, depended on the company requirements and its plan to make use of the advanced technology in its work and improve the way it analyses data and knowledge. Global competitions in the digital age require companies to use technology in managing their work to the maximum extent. With the easing of routine work on database administrators, they can now take advantage of big data, the Internet of things and machine learning in the work of advanced analytics and access to distinct opportunities for the company that can benefit from it in competition and excellence.

DBAs with their new position aim to provide unexpected opportunities for the organization's leaders to enable them to compete and rise to the top. They have to analyze data from past and present trends, discovering and predicting future opportunities (Aleryani, 2020).

In conclusion, the success of the database migration project to the cloud depends to a large extent on the qualification and training of the database administrator on the requirements of migrating to the cloud, understanding the advantages and the risks.

\section{Future studies}

1. Studying and examining the transition of the job of database administrators to the cloud and not being linked to a single business.

2. Use SWOT analysis to evaluate the company's readiness to migrate its database to the cloud.

3. Studying the new role for database administrator as "Data Scientist" and the ability to provide new opportunities for companies using advanced technology regards data analytics.

\section{REFERENCES}

[1] Alam, M. and Shakil, K. (2013). Cloud Database Management System Architecture. UACEE International Journal of Computer Science and its Application Volume 3 Issue 1.

[2] Al-Shafi, A. \& Bahar, A. (2016). Cloud Computing: An Aspect of Information System. International Journal of Applied Information Systems (IJAIS). Foundation of Computer Science FCS, New York, USA Volume 10 - No.4, January 2016. DOI: 10.5120/ijais2016451487

[3] Al Shehri, W. (2013). Cloud Database - Database As A Service. International Journal of Database Management Systems (IJDMS) Vol.5, No.2, April 2013. http://dx.doi.org/10.5121/ijdms.2013.5201

[4] Aleryani, A. (2020). A data analysis perspective by the Business Analyst and Data Scientist Comparative study. International Journal of Scientific and Research Publications, Volume 10, Issue. DOI: 10.29322/IJSRP.10.09.2020.p10525

[5] Bhatti, H. \& Rad, B. (2017). Databases in Cloud Computing: A Literature Review. I.J. Information Technology and Computer Science, 2017, 4, 9-17. DOI: $10.5815 /$ ijitcs.2017.04.02 
[6] Burnham, K. (2018). How Database Administrators Can Keep (or Improve) Their Jobs in the Cloud Era, CIO from IDG. accessed on 3-6-2021 via https://www.cio.com/article/3279960/how-database-administrators-can-keep-or-improve-their-jobs-in-the-cloud-era.html

[7] Chaubey, C. and Nanda, M. (2020). Cloud Database Management System Architecture. International Journal of Electrical Engineering and Technology, 11(10), 2020, pp. 260-266. DOI: 10.34218/IJEET.11.10.2020.036

[8] Cloud Industry Forum (n. d.). 8 criteria to ensure you select the right cloud service provider. Available via https://www.cloudindustryforum.org/content/8-criteriaensure-you-select-right-cloud-service-provider

[9] DeFranzo, S. (2011). What's the difference between qualitative and quantitative research? Available via https://www.snapsurveys.com/blog/qualitative-vsquantitative-research/

[10] HRSA, Health Resources and Services Administration (2011). Readiness Assessment \& Developing Project Aims, Available via https://www.hrsa.gov/sites/default/files/quality/toolbox/508pdfs/readinessassessment.pdf

[11] Kelly, K. (2020). The Changing Role of the Database Administrator (DBA). Dice Accessed on 25-04-2-21 via https://insights.dice.com/2020/03/26/changing-roledatabase-administrator-dba/

[12] Malliga, P. (2012). Database Services for Cloud Computing - An Overview. International Journal Of Computers \&Amp; TECHNOLOGY, 2(3), 67-70. https://doi.org/10.24297/ijct.v2i3a.2674

[13] Marvin, R. (2017). AI Databases: What They Are and Why Your Business Should Care, PC accessed on 30 June 2021 via https://www.pcmag.com/news/aidatabases-what-they-are-and-why-your-business-should-care

[14] McKendrick, J. (2019). The Changing Role of the DBA in the Expanding Cloud World. Big Data Quarterly BDQ accessed on 23-04-2021, via https://www.dbta.com/BigDataQuarterly/Articles/The-Changing-Role-of-the-DBA-in-the-Expanding-Cloud-World131122.aspx\#: :text=Moving\% 20to\%20a\%20cloud\%20data,the\%20dawn\%20of\%20relational\%20databases.

[15] Mew, L., Money, W.H. (2018). Cloud Computing: Implications for Information Systems Development Service Providers and Practitioners. Journal of Information Systems Applied Research, 11(3) pp 35-47.

[16] Neu, W., Vlasceanu, V., Oram, A., and Alapati, S. (2019). O'Reilly Media, Inc., 1005 Gravenstein Highway North, Sebastopol, CA 95472.

[17] Norris, D. (2017a). The Cloud DBA: Role, Activities, Salary. Stratoscale. access on 25- 04-2021 via https://www.stratoscale.com/blog/dbaas/cloud-dba-roleactivities-salary/

[18] Norris, D. (2017b). 5 DBA Skills Enhanced by the Cloud Realm. Stratoscale. accessed on 25-04-2021 via https://www.stratoscale.com/blog/dbaas/5-dba-skillsenhanced-cloud-realm/

[19] Radack, S. (2012). Cloud Computing: A Review of Features, Benefits, and Risks, and Recommendations for Secure, Efficient Implementations, ITL Bulletin, National Institute of Standards and Technology, Gaithersburg, MD.

[20] SADA.com (2019). 9 Steps for Migrating Databases to the Cloud, accessed on 1-6-2021 via https://sada.com/insights/blog/9-steps-for-migrating-databases-to-thecloud/\#: :text=Migrating\%20your\%20databases\%20to\%20the,duplicative\%2C\%20incomplete\%2C\%20or\%20corrupted.

[21] Sawas, M. \& Watfa, M. (2015). The Impact of Cloud Computing on Information Systems Agility. Australasian Journal of Information Systems, 2015, vol. 19, pp. 97-112

[22] Smith, S. (2015). 6 Technologies On Track To Improve Data Integration, database, trends and applications, accessed on 30 June 2021 via https://www.dbta.com/Editorial/Trends-and-Applications/6-Technologies-On-Track-To-Improve-Data-Integration-104229.aspx

[23] Suttle, R. (n. d). SWOT Analysis for an Employee. CHRON, accessed by 15-07-2021 via https://smallbusiness.chron.com/swot-analysis-employee-3340.html

\section{AUTHOR}

First Author - Arwa Aleryani, Associate Prof., arwa.aleryani@gmail.com

Correspondence Author - Arwa Aleryani, arwa.aleryani@gmail.com, dr.aleryaniarwa@gmail.com 